\title{
Aportes de Niklas Luhmann a la teoría de la complejidad
}

\author{
Bernardo A. Castro Sáez \\ Departamento Provincial de Educación de Valdivia, Región de Los Ríos, Chile. \\ Email: bealcasa@gmail.com
}

\begin{abstract}
Resumen: El presente artículo está centrado en la teoría de sistemas de Niklas Luhmann, y su aporte a la teoría de la complejidad. A partir de la necesidad de observar y generar una nueva teoría que pudiese dar cuenta de las diversas relaciones que surgen de los fenómenos sociales, Luhmann trata de construir una teoría, introduciendo diversos conceptos que le permiten reducir la complejidad; pero a la vez, esa misma operacionalización posibilitó en Luhmann una creciente complejización de sus ideas, siendo uno de los primeros intelectuales que se interesó y que aportó significativamente a la teoría de la complejidad.

Palabras clave: complejidad, comunicación, código, paradoja.
\end{abstract}

\section{Contributions of Niklas Luhmann to the theory of complexity}

\begin{abstract}
Abstrac: This article focuses on Nikls Luhmann's systems theory and its contribution to the theory of complexity. From the need to observe and generate a new theory that could allow to show the various relationships that arise from social phenomena, Luhmann tries to build a theory by introducing several concepts that allow him to reduce complexity, but at the same time this operationalization enabled in Luhmann's ideas an increasing complexity, being thus one of the first intellectuals who became interested and contributed significantly to the complexity theroy.
\end{abstract}

Key words: complexity, communication, code, paradox.

\section{Contribuições de Niklas Luhmann à teoria da complexidade}

Resumo: Este artigo enfoca a teoria do sistema de Niklas Luhmann ea sua contribuição para a teoria da complexidade. A partir da necessidade de observar e gerar uma nova teoria que poderia explicar as diversas relações que surgem a partir de fenômenos sociais, Luhmann, tenta construir uma teoria através da introdução de vários conceitos que lhe permitem reduzir a complexidade, mas ao mesmo tempo Luhmann na operacionalização disso fez possível, um crescente complexidade das idéias de Luhman, um dos primeiros intelectuais que se interessou e contribuiu significativamente para a teoria da complexidade.

Palavras-chave: Complexidade, código de comunicação, paradoxo. 


\section{Introducción}

El presente trabajo no tiene la intencionalidad de señalar, la evolución y desarrollo que tuvo la teoría general de sistema a partir de Ludwig von Bertalanffy ni las incorporaciones posteriores; tampoco abarcar la complejidad misma que significa abordar todos los elementos que la misma teoría implica. Indudablemente, existe el peligro de hacer simple lo complejo, de tal forma que no sea capaz de señalar ni describir, lo que significa introducir y valorar, la utilización de la complejidad en la teoría de Luhmann. Espero, no caer en un reduccionismo simplista al dar cuenta en forma amplia el sentido de la complejidad en la percepción luhmanniana del mundo.

Sin embargo, en este desafío, pretendo centrar la mirada en aquellos elementos de la teoría de Luhmann, que aportaron significativamente, al reconocer la complejidad como elemento fundamental y que a su vez, fundamenta la comprensión de la realidad.

Luhmann es considerado como uno de los más importantes teóricos alemanes y, como señala Izuzquiza (1990) en la introducción del texto de Luhmann Sociedad y Sistemas: la ambición de la teoría; su obra, no resulta sencillo de describir en una breve introducción, los rasgos esenciales de la obra ni las perspectivas que ésta abre para una reflexión que afecta a puntos esenciales de la tradición filosófica clásica.

Rodríguez y Arnold (1999), señalan, lo difícil que es presentar la vasta obra de Luhmann y la complejidad de su pensamiento. La variedad de temas abordados, lo novedoso de sus postulados y de las vinculaciones que se establecen entre conceptos y conocimientos de diversa procedencia, constituyen dificultades para una presentación sistémica del pensamiento del autor. Por otro lado, gran parte de las traducciones disponibles son poco adecuadas.

A las dificultades anteriores, señala Rodríguez y Arnold, cabe agregar que Luhmann es un autor que a su extraordinaria productividad y variedad de intereses, une una enorme erudición que se evidencia en las detalladas referencias a múltiples fuentes que apoyan algún postulado. A esta erudición se agrega, el hecho de que su teoría, a pesar de mantener una unidad teórica básica, se encuentra en constante renovación, incluyendo en su marco conceptual básico nuevos aportes de los más diversos ámbitos del conocimiento.

A partir de lo anterior, se tratará de dar cuenta de cómo la visión de Luhmann ha sido un aporte interesante para el desarrollo de la complejidad.

\section{La complejidad luhmanniana}

El eje central en la teoría de Luhmann, es el reconocimiento del concepto de complejidad; a diferencia de Morin, quien señala que sólo la complejidad permite abordar la complejidad. Luhmann, sin embargo, la 
reduce, la transforma a través de un proceso de racionalización que permita intervenir y entender la realidad de los sistemas sociales. Para ello, elabora una red sistémica del concepto que posibilite justamente ese entender y comprender.

El problema básico para Luhmann, señala Rodríguez y Arnold (1999), es la complejidad. El sistema no se considera ya (como sucede en el modelo parsoniano) algo dado y, por lo tanto, resulta pertinente preguntarse por su función. Esta función, la de construcción del sistema, consiste en la comprensión y reducción de la complejidad del mundo. Luhmann escoge un concepto de complejidad basado en los conceptos de elementos y relación. Un conjunto de elementos es complejo cuando, debido a limitaciones inmanente (esto significa que es inherente a un ser o va unido de un modo inseparable a su esencia, aunque racionalmente puede distinguirse de ella) de la capacidad de relación de los elementos, cada elemento ya no puede relacionarse en cada momento con cada uno de los otros elementos. Con el concepto de "limitación inmanente", se refiere a la complejidad interna de los elementos, la que no está al alcance del sistema, y que al mismo tiempo permite la "capacidad unitaria" de dichos elementos.

La complejidad sistémica nos remite tanto a variables cuantitativas, tales como la cantidad de elementos y sus posibles relaciones, como a la diferenciación horizontal y vertical de un sistema a los modelos y grados de interdependencia entre sus partes y entre éstas y sus entornos, y en consecuencia, un fenómeno relativo. El concepto de complejidad conduce a la obligatoriedad de la selección, la que a su vez nos remite a la contingencia. Quizás, es desde esta observación que Luhmann (2000:10-11) quiere prevenir, cuando señala que: "Entre más complejo es un sistema, más podrá operar con negaciones, ficciones, presuposiciones analíticas o estadísticas que se distancian del mundo tal como es.”

Es decir, la complejidad permite, la abstracción retórica para poder entender lo que no se puede entender en un momento dado y, al no tenerse en cuenta, imposibilita comprender la dimensionalidad de la complejidad en el sistema mismo; pero, el concepto de complejidad remite, también, a una relación donde existe, entre sistema y mundo una posibilitación mutua de sus posibilidades, dice Luhmann (1975a, 207), citado por Rodríguez y Arnold (1999:101): "La complejidad no es, entonces, sólo la cantidad de las relaciones estructuralmente posibles, sino su selectividad; tampoco es sólo un conjunto de conocimientos (empíricamente asegurados) entre las variables tamaño y estructurabilidad, sino la relación entre determinación positiva del tamaño y la determinación negativa del efecto de eliminación de la estructura. La complejidad tiene su unidad, en consecuencia, bajo la forma de una relación: en la relación de la posibilidad recíproca de cantidades de elementos y órdenes reductivas. En cuanto, unidad de su sistema, la complejidad en sí misma es de naturaleza relacional. Se puede hablar de mayor complejidad, en referencia a los sistemas, cuando aumenta la selectividad de las relaciones posibles de acuerdo con el tamaño y la estructura del sistema." 
Por lo tanto, desde esta perspectiva, la complejidad esta fundamentada por las relaciones que se establecen y la consecuencia que deviene en decidir sobre qué decidir y así, seleccionar sobre aquellos efectos de la relación que permitan al sistema, su sustentabilidad. Así, la complejidad justamente comienza a tener sentido cuando el sentido de los proceso se resuelven en las reflexiones observacionales de las relaciones que se establecen en el sistema. A partir de lo que se observa e interpreta (abstracción retórica), el sistema se dinamiza para generar una acción que permita reducir la compleja telaraña de relaciones que surgen de la interacción comunicacional entre dos elementos del sistema y como una onda expansiva exponencialmente se va deslizando por los laberintos de las interpretaciones que surgen de esas relaciones, permitiendo en algún tiempo y espacio indeterminado de sucesos, tomar la decisión de seguir, detenerse o cambiar. Cuando se toma una decisión, ésta incrementa la posibilidad de reducción del tamaño de la estructura (determinada por la funcionalidad de sus relaciones) y el riesgo que ello implica (efecto provocado por la decisión). La complejidad luhmanniana, se centra en tomar decisiones que surgen de las relaciones para tratar de mantener al sistema en equilibrio permanente (equilibrio en la relación que se da entre: creación y destrucción de las relaciones que se dan en el sistema).

Luhmann (1998) señala que, cuando se piensa sobre el concepto de complejidad, dos conceptos diferentes vienen a la mente. El primero se basa en la distinción entre elementos y relaciones. Si tenemos un sistema con un número creciente de elementos, cada vez se hace más difícil interrelacionar cada elementos con los otros. El número de relaciones posibles deviene demasiado grande con respecto a la capacidad de los elementos para establecer relaciones. Podemos encontrar fórmulas matemáticas que calculen el número de relaciones posibles, pero toda operación del sistema que establece una relación tiene que elegir una entre muchas, la complejidad impone selección. Un sistema complejo surge sólo por selección. Esta necesidad de selección cualifica los elementos, es decir, da cualidad a la pura cantidad. La cualidad, en este caso, no es otra cosa que la capacidad selectiva limitada; es la neguentropía comparada con la entropía, que significa que todas las relaciones lógicamente posibles tienen una oportunidad igual de relación.

Según, Rodríguez y Arnold (1999:102), Luhmann distingue dos conceptos diferentes de complejidad:

a) Complejidad basada en la distinción entre elementos y relaciones. Si se tiene un sistema con un número creciente de elementos, se hace cada vez más difícil relacionar cada elemento con cada uno de los demás. La complejidad requiere selección. Así, la misma necesidad de selección califica los elementos dando calidad a la cantidad. La calidad se entiende como la capacidad selectiva limitada. En comparación con la entropía, que significa que todas las relaciones lógicamente posibles tienen la misma probabilidad de ocurrencia, es negantropía. Este concepto se basa en la operación: es la complejidad de las operaciones. 
b) Complejidad basada en la observación. Si un sistema selecciona por sí mismo sus relaciones, es difícil prever qué relaciones seleccionará. Incluso en el caso de que se conozca una selección particular, no es posible deducir a partir de ella las restantes selecciones. Desde esta perspectiva, la complejidad del sistema es una medida de la falta de información, de la redundancia negativa y de la incertidumbre de las conclusiones que pueden obtenerse a partir de las observaciones hechas. Este concepto, que problematiza la observación, nos recuerda la relación entre entropía e información que hacía Wiener (1948:11, citado por los autores).

Luhmann, agregan Rodríguez y Arnold (1999), sostiene que ambos conceptos, basados en la operación o en la observación, requieren selectividad. Complejidad quiere decir que toda operación, sea intencional o no, controlada o no, observada o no, es una selección. Al ser elemento de un sistema, una operación no puede evitar dejar de lado otras posibilidades. Es por esta misma razón que es posible observar una operación, seleccionar un curso particular y excluir los otros. La selectividad forzada es la condición de posibilidad tanto de la operación como de la observación.

La selección es el elemento central y vital en la reducción de la complejidad y en la forma de cómo la complejidad se desenvuelve en la teoría. La complejidad es la motivadora para gatillar los cambios, mediante la generación de nuevas funciones y estructuras que surgen a partir de esta selección. La capacidad dinámica del sistema está dada por la complejidad de la misma en el proceso de las relaciones que se establecen.

En cuanto a la complejidad del mundo, como sistema, Luhmann (1996b), define el concepto de complejidad en términos muy abstracto. Esto puede hacerse directamente en términos de una distinción entre sistema y el entorno y en término del potencial que posee un sistema para la realización. El concepto, entonces, significa un número de posibilidades que se hacen accesibles a través de la formación del sistema. Implica que las condiciones (y en consecuencia los límites) de posibilidad pueden especificarse que, el mundo llega a constituirse según esta manera y también que, el mundo contiene más posibilidades, de modo que en este sentido tiene una estructura abierta. Desde un ángulo diferente, esta relación entre mundo y sistema puede verse como problema de sobrecarga y como una inestabilidad amenazada constantemente. Éste, de hecho, es el enfoque de la teoría funcionalista de sistemas. Desde la perspectiva opuesta, la misma situación aparece como un orden superior, construido por medio de la reducción de la complejidad a través de la construcción de un sistema que, reduzca el problema a una cuestión de selección. Este enfoque posterior es el de la teoría cibernética de sistema. Luhmann (1997a:77) señala que: “...En conceptos de la teoría de la cibernética se podría formular el mismo problema (la complejidad aumentada) en la forma siguiente: toda planificación debe ante todo descomponer la complejidad del sistema en que ella misma tiene lugar y llevarla a la forma de problemas por solucionar, sobre los que hay que decidir con base en planificaciones ulteriores. Este proceso de 
producción de problemas es en relación a la planificación misma un proceso de retroalimentación positiva, es decir, de reforzamiento de la divergencia."

El mundo es abrumadoramente complejo para todo tipo de sistema real, ya sea que consista en unidades físicas o biológicas, de rocas, plantas o animales; sus posibilidades exceden a aquellas a las que puede responder. Un sistema se localiza a sí mismo en un entorno constituido selectivamente y se desintegrará en el caso de una separación entre el entorno y el mundo. Sin embargo, los seres humanos y sólo ellos son conscientes de la complejidad del mundo y, por lo tanto, de la posibilidad de seleccionar su entorno, lo cual formula cuestiones fundamentales de autoconservación.

Aludiendo a la complejidad del mundo, Luhmann (1996a:82) acota que: "un grado alto de complejidad en el mundo supone una multiplicidad de procesos selectivos; el medio por el cual, las selecciones de las diferentes partes se conectan con cada una de las otras. Y solamente se puede garantizar un mundo presente y simultáneo, si la selección puede presentarse no sólo como el resultado de las propias acciones, sino también, como la selectividad de otros que es simultánea y presente o que puede al menos recordarse o anticiparse en el momento presente. En este sentido existe, por un lado, conexiones intrínsecas entre la complejidad del mundo, y por otra lado, procesos regulados socialmente para diferenciar y conectar selecciones múltiples.”

Igualmente en las organizaciones, Luhmann (1997), señala que la complejidad se constituye en los sistemas organizaciones como la relación entre decisiones. Estas relaciones son el primer contenido de la decisión. Es decir, se decide porque se ha decidido o para que se decida. Las decisiones se califican recíprocamente, definen situaciones unas para otras.

La tesis según la cual el entorno para cada sistema es más complejo que el propio sistema, no supone constancia en el desnivel de complejidad; Luhmann (1990:72) señala que "en general, se puede afirmar, por ejemplo, que la evolución sólo es posible si existe la suficiente complejidad de los entornos de sistemas, lo que significa coevolución de sistemas y entorno. Una mayor complejidad en los sistema aparece solamente cuando el entorno no presenta ninguna distribución azarosa, o mejor dicho, cuando está estructurado selectivamente, a su vez, por sistemas en el entorno.”

Así, el concepto de selección va unido inevitablemente al de complejidad, no puede haber complejidad sin reducción, pues, ésta delimita sus fronteras, las reduce o las disminuye. La selección permite la relación dentro del sistema en pos de la toma de decisión más adecuada para el sistema mismo. Por lo tanto, la relación entre complejidad y selección en la cual se basa Luhmann, no es una descripción de estados. Implica ya el tiempo, y se realiza solamente mediante el tiempo y en el tiempo. El tiempo es lo que obliga a la selección en los sistemas complejos, pues si dispusiéramos de un tiempo infinito todo podría concordar con todo. Visto así, el tiempo 
siempre simboliza que si algo determinado sucede, otra cosa también sucede, de tal manera que ninguna operación aislada puede adquirir un pleno control sobre sus condiciones. Se podría decir que la selección es la dinámica de la complejidad.

No solamente la selección se relaciona con la complejidad, sino que ésta es posible en la medida en que el tiempo actúa como un proceso dinámico de interrelaciones recíprocas. El tiempo es el devenir de sucesos de selecciones. Luhmann (1990:119-121) "Si la relativa autonomía temporal de un sistema puede utilizar la dimensión del tiempo para solucionar mejor los propios problemas de la propia complejidad (a diferencia de los problemas en relación con el entorno) y, sobre todo, para aumentar la propia complejidad mediante la aplicación del tiempo. A esto lo llamaremos “temporalización de la complejidad”. La temporalización de la propia complejidad es la adaptación del sistema a la irreversibilidad del tiempo...La temporalización de la complejidad conduce a un orden selectivo de la interrelación de los elementos, que tiene lugar en un sucesión temporal...La temporalización de la complejidad proviene, como ya hemos dicho, de la temporalización de los elementos del sistema. El sistema esta formado por elementos inestables que duran sólo poco tiempo, o que ni siquiera tienen una duración propia, como por ejemplo, las acciones, y que desaparecen ya en el momento de su aparición...En resumen, un sistema lo suficientemente estable consta de elementos inestables; debe su estabilidad a sí mismo y no a sus elementos; se construye a partir de un fundamento no "existente", y justamente en este sentido es un sistema autopoiético.”

Como se puede apreciar, la complejidad permite la existencia del sistema, y permite, además, su comprensión y estudio. La complejidad es la posibilidad de la evolución y. en este sentido, la observación y la diferencia, permiten operacionalizar el proceso de reducción de la complejidad. Este proceso se lleva, en cuanto el tiempo, posibilita la operacionalización y la existencia de la reducción de la complejidad mediante la selección. El tiempo, hace que la contingencia de eventos sea complejo y la selección su reducción.

Así, el sentido de la comunicación se da en la relación entre la complejidad y la contingencia que se encuentra en la selectividad impuesta por la primera y en el peligro de equivocación involucrado en la segunda. En efecto, la complejidad se refiere siempre a una posibilidad de las que puede actualizarse. La contingencia, por su parte, remite al hecho de que las posibilidades experimentadas por el sistema son sólo posibilidades, y por lo tanto, podrían resultar diferentes a lo esperado. Las posibilidades se visibilizan, mediante la relación de contingencias, es decir, en la doble contingencia que se construye en la operacionalidad de la relación.

La doble contingencia se da mediante conciencia o comunicación, pero no en las dos al mismo tiempo. La doble contingencia que subyace a toda comunicación, es supuesto básico de la formación de los sistemas sociales, no de los sistemas psíquicos. Sólo se puede ser objeto de la concien- 
cia, pero no conciencia misma. Los sistemas sólo pueden obtener su autorrelación y su cerrazón (recursividad), o bien mediante conciencia o bien mediante comunicación, pero nunca con ambas cosas a un tiempo. (Luhmann, 1996b)

La doble contingencia como realización práctica conduce a la formación de sistemas sociales: este es el meollo de los sistemas sociales. Bajo la influencia catalítica propia de la doble contingencia y la presión de selección que provoca, surgen límites que no separan ni unen a los individuos, sino que son una zona propia y única del sistema social. Ella no se constituye en el espacio entre los individuos. En esta región de doble contingencia que resuelve la doble contingencia, los individuos son entornos del sistema de la sociedad, desde el entorno, los individuos están en condiciones de irritar a la sociedad, de formularle preguntas capciosas a los sistemas sociales, de ser insidiosos con las normas o con los que hacen de ella una religión civil de control social, de ejecutar comportamientos inmorales sin temer al Leviathán de la anomia, que es una construcción de facto represiva, o de tender artificios reflexivos que lleven a la sociedad a una presión conversacional que pueda producir mutaciones (Robles, 2002).

Para evitar este panorama desolador que provoca la relación de las personas en el sistema social, Luhmann (1998:238-239) dirá que: “en una situación con doble contingencia, -lo cual significa que en ella cada uno de los participantes tiene que hacer depender su conducta ante los otros de que éstos actúen hacia él satisfactoriamente- existe una necesidad apremiante de que se limite el repertorio de posibilidades. Esta situación precaria, inestable y circular de la doble contingencia es lo que provoca la creación de personas; o dicho más precisamente: es ella lo que hace que los partícipes -ocurra lo que ocurra en el plano psíquico- se compongan en el sistema social- o sea, comunicativamente- como personas, y de acuerdo con las circunstancias dosifiquen cuidadosamente las cualidades sorpresivas de su conducta (bien preparándose cuidadosamente de antemano, para así no topar con límites muy estrechos; bien segmentándola, de manera que otras posibilidades puedan ser rechazadas o ignoradas como no pertinentes al rol; o bien, mediante el manejo de formas de sociabilidad -entre ellas el humor-, de modo que sea fácilmente reconocible que la persona misma se retira de ellas y sólo se evidencia su buena educación).”

Pero, no sólo la capacidad de dosificar los impulsos negativos de la persona en la relación de la comunicación, posibilitará disminuir los riesgos de un quiebre o las presiones que de ella surjan; también, la confianza, juega un rol importante en la reducción de la complejidad y, por ende, en la posibilidad de la doble contingencia que posibilita las expectativas, la selección en el sistema y la reproducción de la misma. La doble contingencia en el tratamiento de la complejidad requiere del acoplamiento de sus estructuras (acoplamiento estructural) que permitan, la adecuación y la flexibilidad para tomar decisiones que no desequilibren al sistema. 
Entonces, ¿cómo funciona un sistema? ¿De qué forma se posibilita el acoplamiento estructural en la relación sistémica? Luhmann recurre a la diferencia sistémica. La diferencia de un sistema a otro permite que el sistema social funcione, pues de otra manera habría estancamiento. Pero, para que la diferencia sistémica se haga efectiva, el sistema utiliza, el código.

Cada sistema posee un código binario que lo distingue de los demás sistemas. Así, por ejemplo, el código del sistema político es gobierno/oposición; del sistema económico, dinero/no dinero; del sistema educativo, capaz/incapaz; del sistema moral, bueno/malo.

El código regula e impide, la anarquía y la incomunicación que imposibilitarían las relaciones en el sistema; aún más, el código posibilita acuerdos y confianza operacionales que hacen más efectiva, el acoplamiento estructural.

El código permite levantar mapas del territorio, aunque no es el territorio, lo describe e interpreta, elabora relaciones de diferencias en la contingencia, mientras que el acoplamiento estructural, lo visibiliza en la diferencia. El código, levanta interpretaciones de la realidad, construye diferencia a partir de la dinámica y operacionalidad estructural que permite, la unidad de la diferencia en el sistema.

Luhmann (1991), describe las características del código binario, de la siguiente forma:

- Son construcciones totalizadoras, construcciones de mundo con exigencias de universalidad y sin limitación ontológica. Todo lo que está ausente de su ámbito de relevancia se subordinará a uno u otro valor por la exclusión de una tercera posibilidad.

- La totalización como relación a todo lo que en el código puede ser tratado como información implica una contingencia sin excepciones de todos los fenómenos.

- Los códigos son abstracciones hipotéticas, pues sólo son validos para un determinado uso en el ámbito de la comunicación no escogido por ellos.

• El codificar utiliza y perfecciona una antigua intuición: “contrarium

- La codificación binaria tiene que renunciar a que el valor que orienta el código (verdad, justicia, propiedad, etc.) sirva al mismo tiempo como criterio de selección.

- La diferencia entre código y criterio para la corrección de las operaciones (o entre codificación y programación) posibilita una combinación de clausura y apertura en el mismo sistema. 
El código, en la teoría de Luhmann, es un oscilador binario que le sirve al sistema para operar. No es un esquema de realidad, es únicamente una ficción que le permite al sistema hacer una reducción de complejidad. Sólo con la condición de la apertura a opciones positivas y negativas, un sistema social puede identificarse a sí mismo con un código. Cuando esto sucede, el sistema reconoce todas las operaciones que se orientan por el propio código como propias y otras no. Mediante el código, el sistema se diferencia a sí mismo del entorno y efectúa, su propia cerradura operativa. Así pues, sólo el código, permite al sistema reconocer las operaciones que le son propias y deslindarlas de las operaciones de otros sistemas.

El código asume la contingencia del mundo y, en virtud de su binariedad, impide que el sistema se trabe en el logro de un fin y deje de operar. Esta necesidad de conservación de la autopoiesis es lo que hace que los sistemas de funciones nunca sean sistemas teleológicos (causas finales). Refieren toda operación a su código binario, es decir, una distinción entre dos valores, y con esto se aseguran la posibilidad de pasar de un valor al opuesto. Lo que ha sido fijado como rendimiento escolar aceptable puede servir en una ulterior comunicación para replantear la pregunta de si es aceptable o no. Un código binario mejor/peor en la función del sistema educativo puede servir para que lo que parecía mejor en un momento dado, ante la adquisición de nuevos datos o teorías, sea revisado. De esta manera el código binario mantiene la autopoiesis del sistema.

En sentido estricto, los códigos binarios son formas de dos partes, lo que constituyen en el sistema, lo complejo. No son representaciones de una realidad de los valores que codifican, sino simples reglas de duplicación, una digitalización, es decir, relación de relaciones que complejiza la observación y la selección de lo que se observa. El código define su ámbito de aplicación y para toda información que aparece en este ámbito pone a disposición un correlato negativo, un valor opuesto. Consecuentemente, todo lo comprendido en la forma del código aparece como contingente, como posible de una manera u otra. Esto obliga en la práctica a disponer de un conjunto de reglas decisionales que fijan las condiciones que permiten asociar cada valor con lo correcto o incorrecto. Tales reglas constituyen, los programas.

Además de los códigos, el sistema utiliza la paradoja para generar las relaciones complejas. Si la paradoja reduce la complejidad, la comunicación la admite, la soporta, la encuentra compatible, familiar, de ahí su reiteración, su insistencia, su terquedad, su empecinamiento.

La complejidad permite la paradoja; pero, la paradoja reduce la complejidad. Según Rodríguez de Rivera (2004), la complejidad se construye, como estado de cosas auto-referencial, pues es en la auto-referencia donde determina ella misma los desniveles de complejidad. Esto significa que la complejidad se construye como "paradoja". Paradójicamente, la complejidad es des-paradojizable por su reducción, y simultáneo incremento, de lo que se observa seleccionando (reduciendo) en diferencia a la delimitación 
de las otras posibilidades definidas como no seleccionables. Si bien la teoría de Luhmann está cruzada por paradojas (incluyendo a la modernidad como una de ellas), vale la pena detenerse en aquellas que se fundamentan en el propio sistema que hace distinciones, o sea, un sistema observador.

El sistema utiliza la paradoja para desenredar aquellas interpretaciones y observaciones que de otra forma no se pueden explicar o resolver. Es un mecanismo de auxilio que el sistema comunicacional utiliza. No es un elemento metafórico o lingüístico. Es un proceso operacional que permite explicar, las decisiones y las acciones que surgen y se dan, en las relaciones que se establecen en el sistema. En la complejidad subyace la paradoja. La paradoja gatilla y motiva una selección y en consecuencia, reducción e incremento de las relaciones que se establecen en la selección de esa misma relación.

La paradoja reduce la complejidad. “..., la complejidad solo podrá reducirse en tanto se dé una mayor complejidad. Sólo el aumento de complejidad puede llevar a una reducción de la complejidad” (Luhmann, 1990:17). La sobreabundancia de complejidad, el aumento de las posibilidades de relación de un sistema, posibilita la reducción de la misma, la paradoja permite en el aumento, la disminución y, a su vez, nuevamente la superabundancia de relaciones que autorreferencialmente vuelve a reducir la paradoja. La paradoja para Luhmann es la posibilidad de romper con la tautología, haciendo que los procesos autorreferente puedan por sí mismo observar y observarse, preguntar y obtener respuesta, ahí donde la lógica deja de tener sentido.

La paradoja, por lo tanto, constituye la sinapsis que permite que, al momento de que el sistema niega la conexión, la conexión entre las relaciones del propio sistema se realiza: clausura y apertura. La comunicación produce la no comunicación en forma de paradoja; pero, al mismo tiempo es la paradoja la que posibilita que esta se comunique, es decir, permite la relación de los proceso al negar, esos mismo procesos de relación. La paradoja, es la herramienta que soluciona aquellos problemas que la racionalidad no puede solucionar. La paradoja, permite la creatividad e innovación, al dar solución a la entropía que presenta el sistema. No es que la paradoja sea en sí misma posibilidad creativa, pero, motiva e impulsa a que el sistema busque mediante la creatividad y la imaginación, una solución para resolver la disyuntiva que le presenta la propia paradoja. De allí, que la paradoja incita al sistema a crear nuevas posibilidades desde su propia negación de posibilidades.

La provocación producida por la paradoja al sistema, hace que ésta reaccione inconscientemente a ese estímulo provocado, resultando una respuesta que no estaba en los procesos racionales de la relación dentro del sistema, es decir, es una respuesta impensada, para las perturbaciones provocadas en el sistema. Son decisiones libres, sin presiones; pero con un alto grado de irracionalidad sistémica, que hace aumentar la complejidad de la misma y al mismo tiempo, reducirla. 
"La paradoja consiste en que las decisiones conscientes se deben tomar inconscientemente -por supuesto, mediante una elección libre y no bajo amenaza o presión, ni tampoco mediante el ofrecimiento de hechos falsos.” Agrega más adelante el autor, “...En todo estos casos, la solución del problema toma forma de paradoja: los individuos, al tomar parte en la comunicación, se individualizan, y se desindividualizan; se uniforman y se ficcionalizan, para que la comunicación pueda proseguir en referencia a los individuos. Y todo esto sin que la operación comunicativa tenga que explicitar que cada individuo es un sistema único operativamente clausurado. La oferta diferenciada de los medios de masas, posibilita que la comunicación social conecte con una permanente referencia a los individuos, sin que se tenga que estar tomando en cuenta la especificidad de los otros sistemas de función” (Luhmann, 2000: 69 y 107-108)

La homogeneidad permite a la paradoja hacerse presente en la comunicación mediante la diferencia, la diferencia posibilita reintroducir la diferencia en lo diferenciado. "De esta forma la diferencia se vuelve al principio paradójica, se convierte en parte de una parte en sí misma. Pero precisamente este rodeo a través de la paradoja de la autorreferencia puede ser fructífero, porque crea un problema permanente para las operaciones del sistema que estimula de una u otra forma la búsqueda de soluciones.” (Luhmann, 1996b:59)

Además, la paradoja se da en la expectativas de un posible futuro promisorio, por ejemplo en el amor, Luhmann manifiesta que: ”...la transición a lo paradójico no significa, en modo alguno, incapacidad de acción. Los amantes no se ven confrontados con modelos "forced choiced” ni con alternativas incompatibles. La paradoja queda referida más bien al plano de las esperanzas depositadas en el compañero en las relaciones íntimas. El amor simboliza que la realización plena de todas esas esperanzas es posible. Las esperanzas normales se filtran por el paso a la paradoja (y no mediante idealización) y así, al mismo tiempo, se prepara el escenario preciso para que el amor pueda aparecer (...). Sólo es posible distanciarse de los ideales mediante una pobre realización y, consecuentemente, los modelos paradójicos estimulan el distanciamiento.” (Luhmann: 1985: 60 y 72).

Además, agrega que: "Se trata de la paradoja de lo que ha sido incluido en el sentido, aparezca como excluido de lo incluido (en el otro)... La misma observación de segundo orden debe presuponer realidad en el observador a quien observa. La observación de segundo orden puede selección al observador, pero no crearlo. Y la razón es simple: cada observación traba con la distinción autorreferencia/heterorreferencia, y esta última debe quedar ocupada. Dicho de otra manera: debe utilizar esta distinción como punto ciego, ya que no puede darse cuenta (no puede observar, ni designar) de que esta distinción es posible gracias a la paradoja de la reentry” (Luhmann, 2000:115 y 130)

La paradoja permite que la observación presuponga en su distinción, una realidad diferenciada. Esta diferenciación, es en sí misma una 
selección de esa realidad diferenciada, pero, no una creación. No se crea la diferencia, la diferencia esta asumida en la selección que realiza el observador en la operacionalidad de la retroalimentación de la información producida por el sistema. De esta forma; el amor, funciona en la relación de la distinción autorreferencia/heterorreferencia, entre lo ideal y la esperanza. Es la ilación entre el presente y el futuro. El futuro en el presente.

“..., la paradoja es una forma de tiempo, que deja abierto el futuro del otro lado de la forma; la paradoja lleva a un nuevo arreglo, a una nueva descripción que tiene a lo usual por cuestionable. Como en la autopoiesis no existe una forma lineal que se manifieste como principio o como fin, entonces no tiene cabida las preguntas por lo "anterior" y por "lo que habrá de venir". Se pueden presentar ciertas propuestas, pero, si se quiere ser coherente con la observación de segundo orden, estas propuestas no son sino estímulos mentales. El fin principal debería ser enseñar a los clientes que en toda distinción se encuentra una paradoja y que la observación sólo es posible cuando las paradojas se presentan en la forma de una distinción que momentáneamente parece convencer.” (Luhmann, 2000:173)

Así, la paradoja es la posibilidad de lo irracional en las relaciones racionales, permitiendo la solución inesperada, la sinapsis precisa y coherente de los contrarios. La paradoja, se da precisamente en la diferencia, que gatilla estímulos en el sistema para las soluciones que de otra forma sería imposible de lograr. Sólo, mediante la paradoja, el sistema consigue desenredar el nudo ciego que surge de la complejidad sistémica y, por supuesto, permite reducir, la complejidad surgida.

Por último; señalaremos, que todos los conceptos que han dado forma a la teoría de Niklas Luhmann, han crecido bajo el tema central y neurálgico tratado por el autor: La comunicación. Permitiendo que los diversos conceptos mencionados, se relacionen mutuamente. No es una cadena de ideas y conceptos entrelazados linealmente, sino que, creativamente e innovadoramente. Éstos surgen en la medida que la teoría trata de aclarar su propia construcción teórica. Concepciones que fundamenta la importancia que tiene, la comunicación en la sociedad, desde la teoría general de sistema. En este sentido, la teoría está ligada íntimamente a la complejidad, razón de ser de la paradoja, que vive y se alimenta de los códigos que surge de las relaciones y que en definitiva, permiten tomar decisiones y seleccionar.

En la teoría de Luhmann, la comunicación es una operación, presupone la participación en la misma de varios sistemas de conciencia, pero como unidad no puede ser imputada a una conciencia en particular. Ahora, la comunicación sólo tiene lugar cuando la selectividad de una notificación es entendida y puede ser usada para la selección de un estado propio del sistema. Todo opera en la comunicación. La comunicación tiene sentido en la compresión del comunicar y en la posibilidad de su selección en el entender de la información que se está comunicando. En este proceso, el có- 
digo cobra relevancia, pero para que esto suceda, el acoplamiento estructural del sistema de conciencia que comunica es fundamental.

El concepto de comunicación es imprescindible para entender la forma que se realiza el contacto entre los sistemas autorreferentes. La comunicación es una operación autopoiética que le permite al sistema realizar tres selecciones distintas: la información, la comunicación y la comprensión. De esta comunicación autopoiética se derivan comunicaciones posteriores para sí y en la relación con el entorno.

Luhmann plantea que, el concepto de comunicación parte de la base de que la comunicación sólo puede producirse cuando el que comprende puede distinguir comunicación e información: “Toda comunicación concreta es una unidad que se deriva de la convergencia de tres selecciones (información, comunicación, comprensión). Para la comprensión de la comprensión, es un momento imprescindible (pero sólo uno de varios) la comprensión de la diferencia entre información y comunicación que lleva a cabo ese entender.” (Luhmann, 1996b:122)

El ser humano no es quien comunica (entendiendo al ser humano como sistema psíquico y orgánico) sino el sistema social. La necesidad de explicar la sociedad sin referencia al sujeto es ciertamente revolucionaria, pues permite desantropologizar a la sociedad. Según, Luhmann, el hecho que se ubique al ser humano en el entorno del sistema social, le da a éste la libertad y complejidad que realmente posee, excluyendo así, la posibilidad que la sociedad esté configurada a imagen y semejanza del ser humano. La configuración de las relaciones del sistema social se da en la comunicación y su diferencia, se manifiesta entre la distinción de informar y comunicar, dando en este proceso, sentido a la comunicación. Entendiendo por "sentido de la comunicación”: hacerse comprender y entender para tomar una decisión.

Así, la improbabilidad se encadena con lo posible o no posible en comunicar algo a alguien, o en no comunicar. Implica en este proceso: el futuro.

"El presente queda referido a un futuro que sólo es dado en el modo de lo probable/improbable. Dicho de otras manera, la forma de futuro es la forma de la probabilidad, que, por su parte, dirige el observar como forma-con-dos-lados: como más o menos probable o como más o menos improbable, los cuales son dos modalidades que se distribuyen sobre todo lo que puede ocurrir. (...) Esto presupone que es posible diferenciar entre el futuro (o el horizonte de futuro) del presente, como ámbito de lo probable/improbable, y los presentes futuros, que serán siempre exactamente tal como serán, y nunca de otro modo" (Luhmann, 1998: 161-162)

Así, la comunicación, no sólo en su proceso de comunicar se vuelve improbable, también, en la selección de decidir, lo que transforma a la co- 
municación, en un proceso donde el riesgo es latente. Con ello, Luhmann resalta el valor que tiene la selección en el modo de comportamiento propio de un sistema social y cómo la selección, si está adecuadamente estructurada, contribuye a reducir eficazmente la complejidad. De fundamental importancia es precisar que la comunicación es propia de los sistemas sociales. Tan sólo, la sociedad y los sistemas sociales comunican, sobre sí mismo y sobre los otros sistemas sociales, guardando los modos propios de la clausura que constituye su autopoiesis. En este sentido, la sociedad se compone de comunicación y es el ámbito de todas las comunicaciones posibles. Pero, a la vez compleja.

Toda comunicación descansa sobre una diferencia bien localizada: precisamente la diferencia entre información y comunicación. Sin la protección de esa diferencia el receptor de la información se vería enfrentado directamente a la información, como en el caso de las percepciones de tipo normal acerca de lo que acontece en el mundo. La comunicación y la aceptación, o en su caso el rechazo, de las selecciones transmitidas a través de esa comunicación, sólo se alcanza cuando el receptor puede diferenciar la selectividad de la información y la selección de la comunicación. Eso significa, también, que tiene que reaccionar frente a ambas y en un momento dado reaccionar de modo diferente ante cada una de ella. Por ejemplo, el receptor de la información puede considerar la comunicación como auténtica y sincera, pero la información (lo informado) como falsa; puede dar las gracias por una noticia desagradable; puede considerar el hecho comunicado como carente de importancia. Si es cierto que esta diferencia entre comunicación e información constituye la unidad comunicativa y que de la capacidad de establecer esa diferenciación depende todo lo que puede obtenerse de la información, la cuestión de las barreras inmanentes de la comunicación debe ser referida precisamente a esa diferencia. (Luhmann, 1985)

"Las informaciones, señala Luhmann, son (y nos mantenemos en ello), distinciones que llevan a efecto una diferencia. Ya el solo concepto presupone una secuencia de por lo menos dos acontecimientos con efecto de marcación. La distinción que es producida como información, puede ser, a su vez, una distinción que provoque una diferencia. Las informaciones, en este sentido, están siempre recursivamente entrelazadas: se dan en relación a otras, pero permiten que se les ordene según una secuencia, en vista de resultados más o menos improbables. Esto puede acontecer en la forma estrecha de un cálculo (o de una operación matemática), pero también en la forma de procesos en los que paso a paso se va obteniendo una información que no estaba contenida allí desde el principio -por consiguiente, que sólo en el resultado del procesamiento de la información se ve qué información es todavía necesaria-." (Luhmann, 2000: 78-79)

La información hace posible la visualización de la comunicación, lo contrario la invisibiliza, imposibilitando la comprensión de la relación en- 
tre los sistemas y su autorreferencia. Es decir, surge la muerte del sistema. La invisibilidad hace que el otro no perciba lo que se comunica y, al no percibirlo, no retribuye a su vez con comunicación, rompiéndose éstas; produciendo la no comunicación y por ende, la muerte de las relaciones entre los sistemas y, entre los elementos del sistema. De allí, que es necesaria la información, porque permite la sobrevivencia del sistema, al comprender sus relaciones en la comunicación.

\section{A modo de conclusión}

El esfuerzo que hace Luhmann, por reducir la complejidad, hace que la misma complejidad cobre, por sí misma, como concepto, una complejización creciente en torno a su misma composición y función dentro de la realidad y, permitió a su vez, la necesidad de observarla y entenderla; no sólo desde su reducción, sino desde su compleja complejidad.

Sin querer, Luhmann, introduce la complejidad en sus observaciones; sin embargo, no se da cuenta que para elaborar una teoría que permita reducir la complejidad, necesariamente tiene que complejizar su propia constructo teórico. La belleza de su elaboración, esta dada por lo flexible y dinámica que resulta su teoría; pues ella, evoluciona con cada elemento nuevo que surge de la información que genera la realidad. No reduce su sentido ni su finalidad a las nuevas concepciones, interpretaciones o paradigmas; más bien, se alimenta de ellas y va mutando su funcionalidad, adecuándose justamente, al entorno de los fenómenos que se van creando a la luz de los nuevos conocimientos y acontecimientos.

La teoría, tiene la capacidad autopoiética de enriquecerse de sus propios elementos que surgen de la comunicación de los sistemas sociales; y ese proceso, paradójicamente no lo hace menos complejo sino todo lo contrario, con mayor riqueza de información y más capacidad de resolver sus propios nudo críticos.

El sistema social de Luhmann, se dinamiza a partir de las relaciones que se establecen en el sistema y que por su exponencial dinámica, se vuelve compleja en su observar. Este proceso de relaciones se produce en el flujo dinámico de la comunicación, para comprender esta operacionalidad, Luhmann recurre a la selección como mecanismo y posibilidad de reducción de la complejidad que paradojalmente, la incrementa. La selección que deviene de la toma de decisión de lo que se observa en las relaciones que se producen en el sistema, se hace a través del tiempo, permitiendo que esta selección ocurra en un devenir de sucesos de relaciones que sería imposible que ocurra fuera de ella. El tiempo, permite la doble contingencia en el sistema y esto sucede, gracias a la capacidad autopiética que tiene el sistema, generando el acoplamiento estructural que proceso necesario, donde la relaciones se suceden fluidamente. En este sentido, para reducir la complejidad, surge la diferencia que se visibiliza mediante códigos binarios, permitiendo la posibilidad de la selección en la comunicación del sistema 
social. Este proceso teórico, construido por Niklas Luhmann es admirable. Sólo se entiende en la medida que, la complejidad es la esencia que posibilita la existencia del sistema social luhmanniano, donde el hombre, está dado en su entorno.

No cabe duda; Luhmann, al querer simplificar el entramado que observaba de la complejidad, necesariamente tuvo que ocuparse de ella. 


\section{Bibliografía}

Izuzquiza, Ignacio (1990), Introducción al texto de Niklas Luhmann Sociedad y sistema. La ambición de la teoría, Ed. Paidós/I. C. E. . A. B., $1^{\circ}$ ed.

Luhmann, Niklas (1985), El Amor como pasión, Ediciones Península, $1^{\circ}$ ed., Barcelona.

Ídem (1990), Sociedad y sistema: la ambición de la teoría, Ediciones Paidós Ibérica, S. A. $1^{\circ}$ ed., España.

Ídem (1991), Sistemas sociales, Ed. Universidad Iberoamericana/Alianza, México.

Ídem (1996a), Confianza, Ed. Anthropos, $1^{\circ}$ ed., España.

Ídem (1996b), Teoría de la sociedad y pedagogía, Ed. Paidós Educador, $1^{\text { }}$ ed., España.

Ídem (1997), Organización y decisión. Autopoiesis, acción y entendimiento comunicativo, Ed. Anthropos. Universidad Iberoamericana, $1^{\circ}$ ed., España.

Ídem (1998), Complejidad y modernidad. De la unidad a la diferencia, Editorial Trotta, España.

Ídem (2000), La Realidad de los medios de masas, Anthropos Editorial, Universidad Iberoamericana, $1^{\circ}$ ed., España.

Robles, Fernando (2002), “Sistema de interacción, doble contingencia y autopoiesis indexical” en: Cinta de Moebio N ${ }^{\circ} 15$, diciembre, Facultad de Ciencias Sociales, Universidad de Chile, www.moebio.uchile.cl/15/ frames04.htm

Rodríguez de Rivera, José (2004), Epistemología de la complejidad, en: CEPADE. Universidad Politécnica de Madrid / Dpto. Ciencias Empresarial, Universidad de Alcalá de Henares. www.sunnuc.cepade.es/jrivera/ bases_teor/episteme/epis_complex/epistem_complex.htm.

Rodríguez, Darío y Arnold, Marcelo (1999), Sociedad y teoría de sistemas, Editorial universitaria. $3^{\circ}$ ed., septiembre, Santiago de Chile.

Recibido: 30.06.2010

Aceptado: 17.03.2011 